\title{
Study on the Appearance and Shape Design of China Pavilion
}

\author{
Dan Chang \\ Changchun Sci-Tech University, Jilin, Changchun, 130600
}

Keywords: China Pavilion, Green, Traditional Elements, Appearance Form

\begin{abstract}
China Pavilion appearance design is the wisdom of Chinese people and the crystallization of power, to mention the Chinese culture and national spirit. China Pavilion design has become a model of the Chinese people's minds, from the appearance of the form to the overall color contains the traditional Chinese atmosphere, deeply felt the ancient Chinese history and culture. China Pavilion's environmental design is also closely followed by the times. China Pavilion design is successful, is the world are praised for the architectural design.
\end{abstract}

\section{Introduction}

The appearance of the Chinese Pavilion design uses the combination of traditional Chinese elements and modern design style, and the use of advanced science and technology. From the color to the design are bold use of the Chinese red color, coupled with green, energy-efficient facilities clever design, so that the Chinese Pavilion design so far intriguing. The design of the China Pavilion has attracted the attention of many people, with bold colors, unique and authentic Chinese Pavilion, not only let the Chinese lament its grandeur, but also let the world recognize the essence of Chinese traditional culture.

\section{The Application of Chinese Red}

When it comes to red, it makes people think of a lot of things related to red, such as: leaves, sun, songs, sunset. Red in every Chinese people's minds mean auspicious, rich, festive. Red is also the color of every Chinese people, but also the color of the Chinese flag. "Chinese Red" symbolizes the unity, progress, enthusiasm of the national character. Chinese Museum of red through the reflection of the sun, so that the whole body has a different response to the visual effects. From top to bottom, from deep to shallow, full of Chinese red color, that is calm and classic color. Museum body with a metal material and corduroy-like texture program, can reflect the sun during the day, even in the night light will also be issued under the golden light, like to put on the body wearing a "red coat", gorgeous present in front, It jumps over the red is more atmospheric.

A red pillar like the shape of the Temple of Heaven, scrambling to stand there, layers of stacked, one after another. Meaning the rapid development of China, the prosperity and prosperity of the motherland. "Chinese red" this concept can be described as leaching, not only the appearance of the Chinese Pavilion, the design in the exhibition hall did not forget it. Some of the exhibition areas within the exhibition hall also use the "Chinese red". Can be described as everywhere, no matter where you go to the exhibition hall where you can see China's use of advanced technology developed from the "Chinese red" color, this color has become China's logo color.

\section{The Full Flavor of the Chinese Pavilion}

China Pavilion concept is "the highest in the East, the peak of China, the world granary, rich people." The overall design takes into account the ancient Chinese Xia and Shang dynasties "tripod", after refining the integrated design appears to see the architectural form. Four pillars, is the characteristics of the tripod, the Chinese Pavilion of the four base corresponds to, is to consider the 
Ding's own design form. This design makes the Chinese Pavilion more unrestrained, symbolizing the authority and strength. The top of the design using a square red square row, like the ancient window grille. The middle part of the museum from the bottom up, is layers of brackets, giving the traditional Chinese architectural elements of the shape of the "fighting", reflecting a very strong Chinese characteristics and temperament. Giant pillars and brackets clever combination, constitute the shape of the inverted trapezoid, taking into account the principles of architectural mechanics, but also a huge challenge building.

China Pavilion is divided into two parts: the National Museum and the Museum, the two venues echo each other, you have me, I have you, are indispensable. In the design of a reasonable layout, clever, there are times, but also a symbol of national unity of the spirit. The regional pavilion has a traditional Chinese flavor from the wall to the garden. The walls are designed with Zhuan text to convey the ancient Chinese twenty-four solar terms. Pavilion platform called "new Jiuzhou Qingyan" city garden, quoted the Yuanmingyuan "Jiuzhou Qingyan". The design of the garden also incorporates the concept of China's Jiangnan Water Forest. China Pavilion of the rail car will be people into the dream of looking for a trip. Part of the design of the exhibition hall using the traditional Chinese architectural style, red square wood as the main design framework, horizontal and vertical interspersed, layers of sets. Quoted the effect of neon lights, constantly changing the color of light and shadow, passing a layer of fog-like story staged in the scene. Greeted the scene is a surprise, as through the development of China's long river. Lattice blocks of white trees, mysterious green transform, as if into the world of the Wizard of Oz. Blue sky, blue river, stands on both sides of the road, call real call, like a tree, so that people pray ... ... Pavilion integration of many elements of traditional Chinese show no doubt, the use of modern design style And the integration of traditional elements, so that the whole building majestic spectacular, magnificent, with a Chinese architectural style. Had to sigh the creativity of the designers, the Chinese traditional elements and modern technology integration of leaching, to create such a wonderful visual feast. While lamenting the magnanimous history of China; sigh modern advanced technology; lament the crystallization of people's wisdom.

China Pavilion night is also very colorful, with four pillars as the center, slowly spread the design of light. The light from the bottom of the middle from the bottom up and outside the four pillars also set the lighting. China Pavilion overall lighting design is the use of LED products to ensure that the "energy saving" requirements, the use of advanced energy-saving lighting technology. But also highlights the stunning and magnificent of the China Pavilion, adding a unique visual effects to enjoy.

\section{The Use of Green and Advanced Materials}

"Green, environmentally friendly" theme, also considered in the China Pavilion, the use of advanced environmental technology to build the museum facilities, such as doors and windows using low-E coated glass, can reduce energy consumption, heat reflection. The top of the design and environmental theme echo, collecting the rain falling in the sky, through the purification system to purify the rain, can be used in the bathroom and vehicle cleaning, such environmental considerations, can be described as amazing!

China Museum of red wood flooring, using a new type of environmentally friendly materials made of wood. Plastic wood is the use of plastic and natural fibers, and the use of a special process and made of wood texture above, there are wood texture. The biggest advantage of plastic wood is to save the wood, to protect the ecological environment. Redwood flooring is not using any paint, and not afraid of water, can not be used after the second, to avoid the pollution of the environment. The use of plastic wood is also three times the average wood. The combination of these advanced technologies also allows the world to see the development of China's economy and the leading level of science and technology to be able to produce such advanced flooring materials, it is important that the green, no environmental pollution, consider so thoroughly.

China Pavilion appearance design is indeed surprising, so that the world infected with the red wave. This architectural design is a lot of people hard work and hard work, but also the wisdom of 
the designers of the crystallization. Now the lack of resources available, low-carbon design is now the leading. China Pavilion to do this, whether it is the venue, the appearance of shape design are considered advanced environmentally friendly materials, the museum design also uses a lot of energy-saving technology. This is also a wake-up of the world's countries and people use limited resources is limited, energy conservation, protection of the ecological environment, green low-carbon design awareness. China Pavilion building design is a pioneer of low-carbon building technology, but also the history of Chinese architecture has a pride.

\section{References}

[1] China 2010 Shanghai World Expo official website 